\title{
Archival Protection of Minority Traditional Sports in Yunnan*
}

Yi Yong-jun

School of Physical Education, Yunnan Normal University, Kunming 650500, China

Zi Zhi-yue

Yunnan Minzu University, Kunming 650500, China

Lv Yun, Xu Chang-hong

School of Physical Education, Yunnan Normal University, Kunming 650500, China

\begin{abstract}
In the long history of development, the minority nationalities in Yunnan have created the unique and colorful minority traditional sports, which has greatly enriched the cultural life of the various minorities. However, minority traditional sports in Yunnan have suffered an unprecedented impact due to the influence of modernization and globalization. If they are not timely rescued and protected, these sports will be lost or extinct. In this paper, the necessity of the archival protection of minority traditional sports in Yunnan has been discussed and the measures of archival protection have been also proposed.
\end{abstract}

KEYWORD: traditional sports; archival protection; minority

In the long process of life and production, the various minorities in Yunnan have created the colorful and diverse traditional sports with rich geographical characteristics. They are national, original, competitive, recreational and popular, which have the broad mass base and the rich cultural heritage. These traditional sports have been considered as the major parts of Chinese culture. However, with the rapid economic and social development and the continuous improvement of people's living standards in minority areas in Yunnan, the survival and the development of minority traditional sports have faced with severe challenges. Therefore, how to conduct the effective protection and inheritance has become a very urgent task.

\section{MINORITY TRADITIONAL SPORTS IN YUNNAN}

The minority traditional sports in Yunnan refer to the various physical activities that are gradually created and developed in the long-term production process of minority nationalities. They have the following characteristics: unique national personality, distinct regional feature, long historical tradition and the role of physical fitness and entertainment[1]. The colorful minority traditional sports have a long history. They are developed in different natural environment and social environment, which can reflect the history, the culture, the religion and the customs of each minority. In some ways, they can also show the courage, the wisdom and the pursuit of minority nationalities. These sports are considered as the main ways of physical fitness for minority peoples. At the same time, they are also the effective methods to entertain, communicate with others and beautify the life.

The minority traditional sports in Yunnan are numerous and wide-ranging. According to statistics, the number of traditional sports that are sorted out has been up to 400 , accounting for over $40 \%$ of the items listed in "Chinese Traditional Sports Blog"[2]. Each minority in Yunnan has their own traditional sports, such as the horse racing, the bullfighting, the wrestling and the archery of Yi nationality; the rattle stick dance, the torch festival and the Raosanling festival of Bai nationality; the polishing swing custom, the wrestling, the dancing, the beating spinning top and the hunting of Hani nationality; the dragon boat race, making sandcastles and the elephant-foot drum of Dai nationality; the Dongba dance of Naxi nationality; the crossbow competitions, the climbing arbor and the blowing bamboo of Lisu nationality; the cross-bow shooting, the wooden drum dance, the spring dance and the Jizong top of Wa nationality; the Lusheng dance of Lahu nationality; the knife dance of Jingpo

\footnotetext{
* About the author: Yi Yong-jun, Research Direction: Sports education and training.

Stage Results of Yunnan Education Project (2013Y409).
} 
nationality; the Guozhuang dance of Tibetan nationality.

\section{NECESSITY AND FEASIBILITY OF THE ARCHIVAL PROTECTION OF MINORITY TRADITIONAL SPORTS IN YUNNAN}

\subsection{Urgency of the protection of minority traditional sports in Yunnan}

The minority traditional sports in Yunnan are the traditional cultures that are created and accumulated from generation to generation. They are closely related to the history, the social characteristics, the economic life, the rituals and the customs of each minority. These sports are the crystallizations of the wisdom of all ethnic groups and they are considered as the "living cultural heritage". The protection of the minority traditional sports in Yunnan is not only the foundation of the cultural heritage and the development of each minority, but also an important part to maintain the cultural diversity of China or even the world. With the rapid development of globalization and modernization, the social economy, the transportation and the communication in minority regions of Yunnan have been also greatly developed. The lifestyles and the values of people have been gradually changed. Some excellent traditional cultures are facing the danger of disappearing.

Due to the economic transition and the changes of lifestyle and production, the minority traditional sports that are created in agricultural economy and specific cultural as well as ecological environment have gradually lost the soil of survival and development[3]. Therefore, the protection of the minority traditional sports in Yunnan is of great urgency, which is not only helpful to promote the national spirit and enhance the national pride, but also conducive to the maintenance of ethnic cultural diversity and the promotion of national unity and social harmony.

\subsection{Archival protection is an important way to rescue the minority traditional sports in Yunnan}

There are two ways to protect the minority traditional sports in Yunnan: the first one refers to inheritance. This is the fundamental method to inherit the minority traditional sports. However, it is also considered as the most difficult protection form. The minority traditional sports may be lost due to the death of the inheritors. At the same time, these traditional sports may be disappeared because of the changes of cultural and ecological environment. The second one refers to the archival protection. The archival protection of minority traditional sports in Yunnan is the method that transforms the minority traditional sports into the tangible sports archives through written records, photos, audio, video and digital ways, etc. Afterwards, the people can understand the knowledge and recognize their value through the development of the information resources implied in minority traditional sports archives. Then, they will participate in the activities of protection of minority traditional sports. Ultimately, the minority traditional sports in Yunnan can be effectively protected, inherited and developed.

The archival protection of minority traditional sports in Yunnan is not simply to establish the traditional sports archives, but throughout the whole protection process, including the collection, the collation, the storage and the exploitation of minority traditional sports archives. The minority traditional sports archives can retain the memories of national cultural heritage. Even if the inheritors and the foundation of survival and development are lost, people can still inquire the previous brilliant footprints through the files. They can be immediately recovered if necessary. Therefore, the archival protection is a better way to rescue the minority traditional sports in Yunnan at present[4].

\subsection{Feasibility of the archival protection of minority traditional sports in Yunnan}

The minority traditional sports in Yunnan are the national cultural heritages that are created, developed and inherited by various minorities. The protection measures should follow the relevant laws and regulations of intangible cultural heritage. In the Article II of "Convention for the Safeguarding of Intangible Cultural Heritage", "protection" means to take measures to ensure the viability of the intangible cultural heritage, including the identification, the documentation, the research, the preservation and the protection of the heritage. In the Article III of "Intangible Cultural Heritage Law of the People's Republic of China", it is recorded that the state should save the intangible cultural heritage through identification, recording, filing and other measures. In Article VIII, it is ruled that the cultural departments should fully understand the intangible cultural heritages and establish the archives as well as the related databases of intangible cultural heritage. In the Article XV of "Sports Law of People's Republic of China", it is ruled that the state should encourage and support the excavation, the organization and the improvement of folk traditional sports. In "Eleventh Five-Year Plan" of Sports career, it has been clearly proposed the implementation of "Protection Project of National Traditional Sports. The ongoing folk traditional sports information and the information that is on the verge of disappearing or have disappeared can be retained through collecting objects and audio-visual materials. This shows that it is feasible to take the 
measure of archival protection from the level of national laws and regulations.

During 1985 to 1990 , China has organized the massive excavation and organization work in order to rescue the minority sports heritage scattered in our society. The number of the collected national traditional sports is up to 977 , in which the number of minority sports is 676 and the items of Han nationality account for 301. Afterwards, "Chinese Traditional Sports Blog" has been edited and published[5]. Since the early 2000s, a wave of safeguarding intangible cultural heritage has appeared in whole country. Some precious and endangered folk cultural heritage have been excavated, rescued and sorted out, including a large number of minority traditional sports in Yunnan. In short, through several large-scale rescue operations, a large amount of raw materials of minority traditional sports in Yunnan have been collected, which lays a solid material foundation for establishing the archives of minority traditional sports. As long as the culture department, the archive department and the sports department coordinate and cooperate with each other, the archival protection of the minority traditional sports can be realized through employing the theories and the methods of archival science.

\section{MEASURES OF THE ARCHIVAL PROTECTION OF MINORITY TRADITIONAL SPORTS IN YUNNAN}

\subsection{Enhancing the national culture consciousness}

With the changes of production and lifestyles in minority areas, the destruction of cultural environment and the decline of traditional culture are increasingly serious. The Chinese folk culture which adapts to the traditional agricultural civilization has been greatly affected. The national awareness of minorities has been gradually weakened and the national cultural indentify has been faded, which makes the colorful and unique minority traditional sports lost the soil to survive. Therefore, it is necessary to stimulate the pride sense of minorities for the traditional sports so as to promote the national identity and cohesion, which is very important for promoting and inheriting the minority traditional sports. At the same time, it is also required to improve the cultural consciousness of whole society in addition to enhancing the cultural consciousness of cultural holders. Only by achieving the consciousness of whole nation and entire society can the sustainable development of archival protection of minority traditional sports be truly realized.

\subsection{Giving full play to the dominant position of government}

The minority traditional sports in Yunnan are numerous and wide-ranged. Meanwhile, there are also some departments involved in organizing work and the work lasts longer. The collected minority traditional sports information is stored in national department, cultural department, sports department, archives and other units. The centralized custody of minority traditional sports archives in Yunnan can not be realized due to the lack of communication among these departments, which brings some obstacles and restrictions to the effective protection and the resource-sharing of minority traditional sports. Therefore, it is necessary to establish the mechanisms in which the cultural department plays the leading role and other departments participate together. They should co-ordinate with each other and play to their strengths so as to realize the effective protection of minority traditional sports archives in Yunnan.

\subsection{Establishing the complete minority traditional sports archives in Yunnan}

Archiving work plays a fundamental role in the process of protecting the minority traditional sports, which is the prerequisite and the basis to carry out other works. The contents of minority traditional sports in Yunnan are rich and wide-ranged. It is required to adopt the multi-channel working methods in the archive collection process according to the lifestyles, the rituals, the customs and the festivals of minorities. At the same time, the minority traditional sports information can be obtained through census and collection as well as the way directly involved in activities. In order to make the minority traditional sports archives more vivid and lively, it is required to employ the high-tech means and establish the more complete archives after standard operation. The minority traditional archives in Yunnan contain the following aspects: the first one refers to the minority traditional sports archives in Yunnan, including their historical origins, the geographical distribution, the specific content, the forms, the inheritance status and the endangered situation, etc; the second one refers to the archives of inheritors; the third one refers to the specific information of the physical props which are related with the minority traditional sports.

\subsection{Developing the archive resources of minority traditional sports in Yunnan}

The ultimate purpose of protecting the minority traditional sports in Yunnan is to inherit and promote the national culture. Therefore, it is necessary to comply with the principles of "protection and rescue first, rational development 
and inheritance development". At the same time, it is also demanded to greatly develop and use the minority traditional sports archives as well as achieve the sharing of archival resources so that more people can understand their meanings and these resources can be run from generation to generation. Firstly, the protection units of minority traditional sports at all levels in Yunnan should cooperate with each other and present the rescued archives. The forms such as exhibition, lecture, performance and publishing the research results can be adopted so as to enhance the public's understanding and recognition for minority traditional sports in Yunnan. At the same time, people can fully understand the precious values and the unique roles of these resources, which lays the solid ideological foundation and mass basis for the protection of minority traditional sports in Yunnan. Secondly, it is required to dig and study the archival resources of minority traditional sports. Meanwhile, it is also necessary to conduct the industrial development for the traditional sports with economic value. Thirdly, it is demanded to establish the archival database of minority traditional sports and achieve the data management. The data management has some advantages such as the rapid transfer speed and the wide transfer range. People can query and retrieve the digitized archival resources of minority traditional sports through the computers and other technical equipments so as to get rid of the limitations of time and space, which is convenient for people to understand and study the minority traditional sports in Yunnan.

In short, with the social development as well as the continuous changes of natural and human environment in minority areas, there are also some difficulties in the process of the protection of minority traditional sports in Yunnan. Therefore, we should have a high sense of historical responsibility. In view of the urgent demands of rescuing the national heritage, it is necessary to give full play to the dominant position of government, stimulate the cultural consciousness of whole society, establish the minority traditional sports archives, develop and use the minority traditional sports archives and realize the sharing of archival resources so that the effective protection and the permanent inheritance of minority traditional sports in Yunnan can be achieved.

\section{REFERENCES}

[1] Ni Yi-ke. Discussion on Development of Chinese Traditional Ethnic Sports. Journal of Sports and Science No.11. Nov.2004

[2] Rao Yuan, Liu Zhu. National Sports in Yunnan. Kunming: Yunnan People Press, 2000

[3] Ni Yi-ke. Discussion on Protection of National Traditional Sport Culture Heritage. Journal of Sports and Science No.8. Aug.2006

[4] Zi Zhi-yue, Xiao Li-yi. The File Protection Study of the Blang nationality intangible cultural heritage. Journal of Lantai World No.7.2012

[5] State Physical Culture and Sports Commission. The Record of Chinese National Traditional Sports. Nanning: Guangxi National Press, 1990

[6] Zhang Hong-yu, Li Xiao-lan. Discussion of the Save and Protect on the National Minority Sports. Journal of Beijing Sport University, No.10.2006

[7] Liu Xiang. Discussion on the characteristic and industrialization of Yunnan National Minority Sports. Journal of Thinking No.6.2004

[8] Chen Wei, Yang Xin-ling, Cai Qi-jiao. Investigating the National Minority Sports Culture Resources in Yunnan. Journal of Sports Research and Education No.5.2012 